Colloq. Algebraic Topology, 1962, pp. 104-113, Matematisk Institut, Aarhus Universitet, Denmark. 310.

4. M. F. Atiyah, Thom complexes, Proc. London Math. Soc. (3) 11 (1961), 291-

5. M. F. Atiyah and J. A. Todd, On complex Stiefel manifolds, Proc. Cambridge Philos. Soc. 56 (1960), 342-353.

6. Sze-Tsen Hu, Homotopy theory, Pure and Applied Mathematics VIII, Academic Press, New York and London, 1959.

7. I. M. James, Spaces associated with Stiefel manifolds, Proc. London Math. Soc. (3) 9 (1959), 115-140.

University of Chicago

\title{
CURVATURE IN NILPOTENT LIE GROUPS
}

JOSEPH A. WOLF

1. Introduction. The purpose of this note is to prove:

TheOREM. Let $M$ be a Riemannian manifold which admits a transitive connected noncommutative nilpotent Lie group of isometries. Given $x \in M$, there exist 2-dimensional subspaces $R, S$ and $T$ of the tangentspace $M_{x}$ such that sectional curvatures satisfy

$$
K(S)<0<K(T) \text { and } K(R)=0 .
$$

Corollary. Let $G$ be a connected nilpotent Lie group, let $B$ be a positive definite symmetric bilinear form on the Lie algebra (\$) of $G$, and let $M$ be the Riemannian manifold obtained by left translation of $B$ to every tangentspace of $G$. Then these are equivalent:

1. $M$ has a positive sectional curvature.

2. $M$ has a negative sectional curvature.

3. $G$ is not commutative.

To prove the Corollary from the Theorem, one simply observes that $M$ must be flat if $G$ is commutative.

The interest of the Theorem and its Corollary is based on the deep similarity between nilpotent Lie groups and Riemannian manifolds of nonpositive sectional curvature. Two striking points of similarity are their coverings (compare [4] with $\$ 4.2$ of [3]) and their exponential mappings. The results of this note show that the class of Riemannian manifolds obtained by placing left invariant metrics on nilpotent Lie groups is quite different from the class of Riemannian manifolds of nonpositive sectional curvature. In the nonflat case one

Received by the editors November 27, 1962. 
expects a manifold of the former class to have a negative sectional curvature, but it is surprising to see that it also has a positive sectional curvature.

Finally we remark that the Theorem does not extend to manifolds admitting a transitive noncommutative connected solvable Lie group of isometries, for the Iwasawa decomposition shows that this class of manifolds includes the irreducible Riemannian symmetric spaces of nonpositive sectional curvature.

2. The positive curvature. The existence of the plane section $T$ of the Theorem will be derived later as a consequence of :

LEMMA 1. Let $G$ be a connected transitive nilpotent Lie group of isometries of a Riemannian manifold $M$ of nonpositive sectional curvature. Then $G$ is commutative.

Proof of Lemma 1. The universal Riemannian covering manifold of $M$ admits a covering group of $G$ as a connected transitive Lie group of isometries [2, proof of Théorème 1]; thus we may assume $M$ simply connected. $M$ is complete by homogeneity so one has the de Rham decomposition; this induces $M=M_{0} \times M^{\prime}$, where $M_{0}$ is a euclidean space and $M^{\prime}$ is a product of irreducible non-euclidean Riemannian manifolds; the latter induces a decomposition $I(M)=I\left(M_{0}\right) \times I\left(M^{\prime}\right)$ of full groups of isometries. Now every element $g \in G$ is of the form $g=\left(g_{0}, g^{\prime}\right)$ with $g_{0} \in I\left(M_{0}\right)$ and $g^{\prime} \in I\left(M^{\prime}\right)$. Let $G_{0}$ be the group generated by the $g_{0}$, let $G^{\prime}$ be the group generated by the $g^{\prime}$, and let $H$ be the closure of $G_{0} \times G^{\prime}$ in $I(M)$.

Let $T$ be the isotropy subgroup of $H$ at $x \in M$. $H$ is transitive on $M$ because $G \subset H$, and $H$ is nilpotent by construction. $T$ is compact because $H$ is closed in $I(M)$, and $T$ is connected because $H$ is connected and $M$ is simply connected, whence $T$ is a torus. Now a glance at the kernel of the universal covering, and the exponential map of the universal covering group, of $H$ shows that $T$ is central in $H$ because $H$ is nilpotent. Thus $T=\{1\}$ because $H$ acts transitively and effectively on $M$. This proves that $H$ is simply transitive on $M$. As $G$ is transitive and $G \subset H$, it follows that $G=H$. We conclude that $G=G_{0} \times G^{\prime}$ as direct product of closed subgroups.

Let $Z$ be the center of $G . Z=Z_{0} \times Z^{\prime}$, where $Z_{0}$ is the center of $G_{0}$ and $Z^{\prime}$ is the center of $G^{\prime}$. An element $z^{\prime} \in Z^{\prime}$ induces an isometry of constant displacement of $M$ because $z^{\prime}$ centralizes a transitive group $G$ of isometries. It follows from the hypothesis that $M$ is of nonpositive sectional curvature [4, Theorem 1$]$ that $z^{\prime}$ acts by a translation along $M_{0}$ and is trivial on $M^{\prime}$. This proves $Z^{\prime}=\{1\}$. As $G^{\prime}$ is nil- 
potent, it follows that $G^{\prime}=\{1\}$. Transitivity of $G$ now implies $M^{\prime}$ $=$ (point). We have proved that $M$ is a euclidean space. Looking at bases, nilpotency of $G$ implies that $G$ is the group of all ordinary translations of $M$. Thus $G$ is commutative. This completes the proof of Lemma 1.

3. The negative curvature. The existence of the plane section $S$ in the Theorem is given by:

LEMMA 2. Let $G$ be a connected noncommutative transitive nilpotent Lie group of isometries of a Riemannian manifold $M$. Then $M$ has a negative sectional curvature.

Proof of Lemma 2. As in Lemma 1, we may assume $M$ to be simply connected, and it then follows that $G$ is simply transitive on $M$. Thus we view $M$ as the Riemannian manifold obtained from $G$ by left translation to every tangentspace of some positive definite symmetric bilinear form $B$ on the Lie algebra $(\$)$.

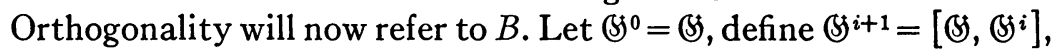
and choose the subspaces $\mathfrak{A}^{i} \subset \mathfrak{S}^{i}$ such that $\mathfrak{S}^{i}=\mathfrak{A}^{i}+\mathfrak{S}^{i+1}$ is an orthogonal direct sum. Let $t$ be the smallest integer such that $\mathfrak{A}^{t}$ has an element which is not central in $\$ S$. We have orthogonal direct sums $\mathbb{B}=\mathfrak{A}^{+}+\mathbb{S}^{t}$ and $\mathbb{S}^{t}=\mathfrak{A}^{t}+\left(\mathfrak{S}^{t+1}, \mathfrak{S}^{t+1} \neq 0\right.$ because $\left(S^{t}\right.$ is not commutative, and $\mathfrak{A}$ is central in $\mathbb{S}$.

Suppose first that $\left[\mathfrak{A}^{t}, \mathfrak{A}^{t}\right] \neq 0$. Then we have an orthonormal basis $\{X, Y\}$ of a plane $S \subset \mathfrak{P}^{t}$ such that $[X, Y]=Z \neq 0$.

$$
X \perp[X, \xi], \quad X \perp[Y, \text { अ] and } Y \perp[X, \xi] \text {. }
$$

For the statements of $\left({ }^{* *}\right)$, one observes that $[X, \xi]$ and $[Y, \xi]$ lie in $\left[\left(s^{t},(s)\right]=\left(s^{t+1}\right.\right.$.

Now suppose that $\left[\mathfrak{A}^{t}, \mathfrak{A}^{t}\right]=0 . \mathfrak{A}^{t}$ has an element $X$ of length 1 which is not central in $\mathfrak{B}$, so $\mathfrak{B}=[X, \mathfrak{S}] \neq 0$. Observe that $\mathfrak{B}=\left[X, \mathfrak{S}^{t+1}\right]$ by choice of $t$ and hypothesis on $\mathfrak{A} t$. Let $x$ be the transformation $W \rightarrow[X, W]$ of $\left(s^{t+1}\right.$ into itself. There is an orthogonal direct sum $\mathfrak{B s}^{t+1}=\mathfrak{U}+\mathfrak{B}$, and $\mathfrak{U}$ has the same dimension as the kernel of $x$. If $x(\mathfrak{U})=0$, then $x: \mathfrak{B} \rightarrow \mathfrak{B}$ would be $1-1$, which is impossible because $\mathfrak{B} \neq 0$ and $x^{\kappa}=0$ for some $\kappa$. Thus $\mathfrak{U}$ has an element $Y$ of length 1 with $[X, Y]=Z \neq 0$. Now observe that

$$
X \perp[X, \xi], \quad X \perp[Y, \mathbb{B}] \text { and } Y \perp[X, \mathbb{B}]
$$

by construction. Let $S$ be the plane spanned by $X$ and $Y$.

We will prove in both cases that the sectional curvature $K(S)<0$. Let $\Omega$ be the curvature tensor of the Levi-Civita connection on $G$. 
As $\{X, Y\}$ is an orthonormal basis of $S$, we have

$$
K(S)=-B(R(X, Y) X, Y) .
$$

Let $\alpha:(\xi \times B) \rightarrow B$ be the connection function of K. Nomizu [1]. In our case, according to Nomizu [1, formula 9.6],

$$
\Re(X, Y) X=\alpha(X, \alpha(Y, X))-\alpha(Y, \alpha(X, X))-\alpha([X, Y], X) .
$$

$\alpha$ is given by $\alpha(V, W)=(1 / 2)[V, W]+U(V, W)$, where

$$
\left({ }^{* * *}\right) B(U(V, W), A)=-(1 / 2)\{B([V, A], W)+B([W, A], V)\} \text {. }
$$

Observe that our formula $(* * *)$ differs from Nomizu's formula 13.1 of [1] by a sign. If the reader follows Nomizu's derivation of $[1$, formula 13.1], he will see that $(* * *)$ is correct and Nomizu's suffers from a misprint.

From $\left({ }^{* *}\right)$ and $\left({ }^{* *}\right)$, we see that $U(X, X)=0$ because $X \perp[X,(\xi)]$ and that $U(X, Y)=U(Y, X)=0$ because $X \perp[Y$, (\$) $]$ and $Y \perp[X, \$)$. It follows that $R(X, Y) X=(1 / 4)[X,[Y, X]]+(1 / 2) U(X ;[Y, X])$ $-(1 / 2)[[X, Y], X]-U([X, Y], X)$. The first and third terms are orthogonal to $Y$ by $\left({ }^{* *}\right)$, and the second and fourth terms total $-(3 / 2) U([X, Y], X)$. Applying $(* * *)$ for the second equality and $0 \neq Z=[X, Y]$ for the final inequality, this gives us $K(S)$ $=(3 / 2) B(U([X, Y], X), Y)=-(3 / 4)\{B([Z, Y], X)+B([X, Y], Z)\}$ $=-(3 / 4) B(Z, Z)<0$. Lemma 2 is proved.

4. Proof of Theorem. Let $G$ be a transitive connected noncommutative nilpotent Lie group of isometries of $M$, and choose $x \in M$. Noncommutativity of $G$ and Lemma 1 show that there is a tangent 2-plane $T^{\prime}$ to $M$ at some point $y$ with $K\left(T^{\prime}\right)>0$. Noncommutativity of $G$ and Lemma 2 provide a tangent 2-plane $S^{\prime}$ to $M$ at some point $z$ with $K\left(S^{\prime}\right)<0$. Let $T=g_{*}\left(T^{\prime}\right)$ and $S=h_{*}\left(S^{\prime}\right)$ where $g, h \in G$ with $g(y)=h(z)=x$; then $K(S)<0<K(T) . K$ is a continuous function on the Grassmann manifold of tangent 2-planes to $M$ at $x$; it must vanish on some plane $R$. The Theorem is proved.

\section{REFERENCES}

1. K. Nomizu, Invariant affine connections on homogeneous spaces, Amer. J. Math. 76 (1954), 33-65.

2. J. A. Wolf, Sur la classification des variêtés riemanniennes homogènes à courbure constante, C. R. Acad. Sci. Paris 250 (1960), 3443-3445.

3. - On locally symmetric spaces of non-negative curvature and certain other locally homogeneous spaces, Comment. Math. Helv. 37 (1963), 266-295.

4. - Homogeneity and bounded isometries in manifolds of negative curvature, Illinois J. Math. 8 (1964), 14-18.

University of California, Berkeley 